\title{
Affective and emotional dysregulation as pre-dementia risk markers: exploring the mild behavioral impairment symptoms of depression, anxiety, irritability, and euphoria
}

\author{
Zahinoor Ismail,, 1,2 Jennifer Gatchel, 3,4 Daniel R. Bateman, 5,6 \\ Ricardo Barcelos-Ferreira, ${ }^{7}$ Marc Cantillon, ${ }^{8}$ Judith Jaeger, ${ }^{9}$ Nancy J. Donovan ${ }^{10}$ \\ and Moyra E. Mortby ${ }^{11,12}$
}

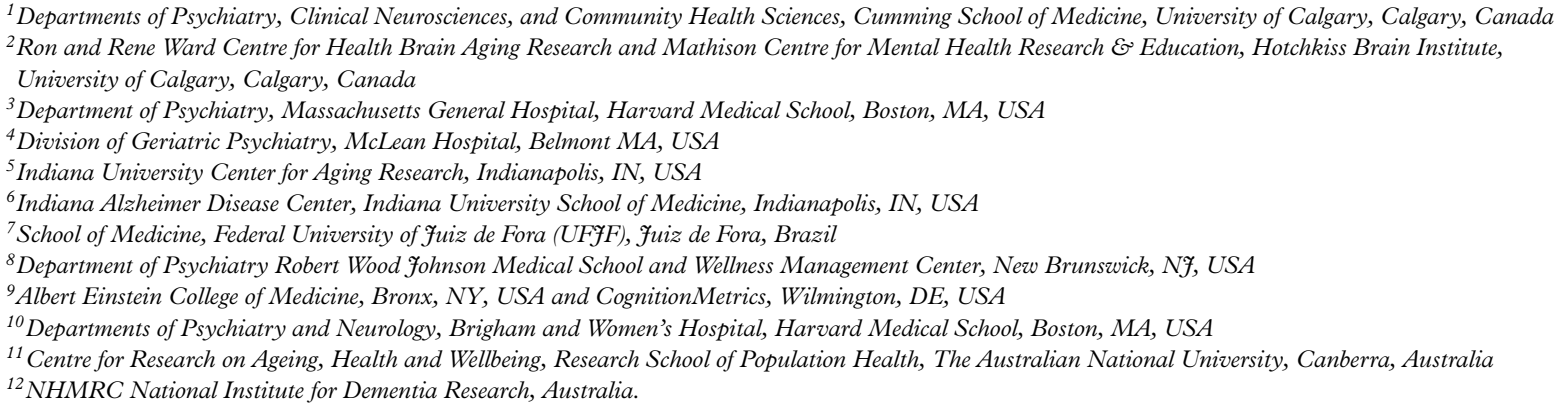

Background: Affective and emotional symptoms such as depression, anxiety, euphoria, and irritability are common neuropsychiatric symptoms (NPS) in pre-dementia and cognitively normal older adults. They comprise a domain of Mild Behavioral Impairment (MBI), which describes their emergence in later life as an at-risk state for cognitive decline and dementia, and as a potential manifestation of prodromal dementia. This selective scoping review explores the epidemiology and neurobiological links between affective and emotional symptoms, and incident cognitive decline, focusing on recent literature in this expanding field of research.

Methods: Existing literature in prodromal and dementia states was reviewed, focusing on epidemiology, and neurobiology. Search terms included: "mild cognitive impairment," "dementia," "prodromal dementia," "preclinical dementia," "Alzheimer's," "depression," "dysphoria," "mania," "euphoria," "bipolar disorder," and "irritability."

Results: Affective and emotional dysregulation are common in preclinical and prodromal dementia syndromes, often being harbingers of neurodegenerative change and progressive cognitive decline. Nosological constraints in distinguishing between pre-existing psychiatric symptomatology and later life acquired NPS limit historical data utility, but emerging research emphasizes the importance of addressing time frames between symptom onset and cognitive decline, and age of symptom onset.

Conclusion: Affective symptoms are of prognostic utility, but interventions to prevent dementia syndromes are limited. Trials need to assess interventions targeting known dementia pathology, toward novel pathology, as well as using psychiatric medications. Research focusing explicitly on later life onset symptomatology will improve our understanding of the neurobiology of NPS and neurodegeneration, enrich the study sample, and inform observational and clinical trial design for prevention and treatment strategies.

Key words: depression, anxiety, euphoria, irritability, affective dysregulation, mild behavioral impairment, neuropsychiatric symptoms, prodromal dementia

Correspondence should be addressed to: Moyra E. Mortby, Centre for Research on Ageing, Health and Wellbeing, Florey Building, 54 Mills Road, The Australian National University, Canberra, ACT 2601, Australia. Phone: +6126158413; Fax: +61261250733. Email: moyra.mortby@anu.edu.au. Received 19 Mar 2017; revision requested 1 May 2017; revised version received 4 Aug 2017; accepted 20 Aug 2017. First published online 13 September 2017. 


\section{Background}

The role of emergent neuropsychiatric symptoms (NPS) as a risk factor for all-cause dementia is increasingly being recognized. Evidence links NPS in advance of dementia to a greater likelihood of cognitive impairment (Mortby et al., 2017) and cognitive decline (Geda et al., 2014) compared to those without NPS. Affective and emotional dysregulation symptoms are among the most common NPS presenting in mild cognitive impairment (MCI) (Peters et al., 2012), and are, along with other NPS, associated with poorer outcomes overall (Cerejeira et al., 2012) and higher conversion rates from MCI to dementia (Palmer et al., 2007).

While the early presentation of NPS in the course of neurodegenerative disease has traditionally raised suspicion of behavioral variant frontotemporal dementia (bvFTD), evidence also suggests that NPS can emerge in advance of any dementia syndrome (Taragano et al., 2009). Historically, these symptoms have been viewed through the lens of psychiatric nosology with insufficient attention given to their late life onset and consequently their frequent misclassification as idiopathic psychiatric illness (Woolley et al., 2011), potentially exposing patients to inappropriate medications or delays in dementia diagnosis (Jalal et al., 2014). Cognitive patterns alone may not adequately differentiate between psychiatric and neurodegenerative disease (Ting et al., 2010).

Mild Behavioral Impairment (MBI) is characterized by later life acquired, sustained and impactful NPS of any severity that cannot be better accounted for by other formal medical and psychiatric nosology. MBI is an "at risk" state for incident cognitive decline and dementia, and for some, MBI is the index manifestation of neurodegeneration, observed in advance of cognitive impairment (Ismail et al., 2016). Importantly, MBI distinguishes between chronic psychiatric symptomatology and formal psychiatric illness, versus new onset psychiatric symptoms in older adults, the latter of which are core to the MBI construct of the at-risk state. MBI has been formally described in the International Society to Advance Alzheimer's Research and Treatment - Alzheimer's Association (ISTAARTAA), MBI proposed research diagnostic criteria, which classify MBI into the domains of decreased drive/motivation, affective/emotional dysregulation, impulse dyscontrol and agitation, social inappropriateness, and delusions and hallucinations (Ismail et al., 2016). Assessment of MBI has been operationalized with the development of the MBI checklist (MBI-C), which was tailored specifically to the MBI criteria, including explicit observations of symptoms being later life in onset, and sustained for 6 months - these requirements are not explicit in many NPS rating scales (Ismail et al., 2017a). The affective/emotional dysregulation domain is a core feature of MBI, and includes symptoms of anxiety, depression and dysphoria, euphoria, and irritability. In this review, we will explore the epidemiology and neurobiological links between affective and emotional symptoms and incident cognitive impairment and all-cause dementia.

\section{Depression and dysphoria}

Increasing evidence in both cognitively normal (CN) older adults (without objective cognitive impairment) and in MCI suggests that emergent depression is a risk factor for dementia (Rosenberg et al., 2013) and may constitute a prodrome (Ismail et al., 2014). This is true for both Alzheimer's disease (AD) dementia and all-cause dementia (Barnes et al., 2012; Steenland et al., 2012; Geda et al., 2014). In a meta-analysis of 57 MCI studies, depression was found to be common, with a prevalence of $25 \%$ in community samples and $40 \%$ in clinical samples (Ismail et al., 2017b), and associated with increased risk of progression to $\mathrm{AD}$ dementia (Hermida et al., 2012; LopezAnton et al., 2015). Another recent study assessed the association of subsyndromal depression (SSD) with cognitive decline over four years in a sample of MCI patients from the Alzheimer's Disease Neuroimaging Initiative (ADNI). Compared with individuals without depressive symptoms, the SSD group exhibited accelerated decline on cognitive measures (Gonzales et al., 2017). This suggests that in early stages of $\mathrm{AD}$, depressive symptoms may be clinical indicators of AD pathology and/or impact AD pathogenesis (Steenland et al., 2012; Donovan et al., 2014; Geda et al., 2014; Gonzales et al., 2017).

Very recent research has moved the field rapidly forward, and underscored the importance of incipient depressive symptoms in emergence of dementia syndromes. A 28-year follow up of a 10,189 person cohort from the United Kingdom described the emergence of sustained depressive symptoms to increase the risk of incident dementia, but longstanding sustained depressive symptoms offered no increased risk (Singh-Manoux et al., 2017). A 14-year longitudinal Australian study of 4,922 cognitively intact men demonstrated that depression is more likely to be a marker of incipient dementia than a truly modifiable risk factor due to a time-dependent link between onset of depression and incidence of dementia. 
The association between depression and dementia was only apparent during the initial five years of follow-up, and was not observed if depression emerged more than five years before the onset of dementia (Almeida et al., 2017). Similarly, a Finnish nationwide nested case-control study of 27,948 pairs also established the importance of the time window between psychiatric symptoms and dementia. Hospital treated behavioral and mental disorders, including depression and other mood disorders, were associated with a higher risk of $\mathrm{AD}$ in the five-year but not the tenyear time window (Tapiainen et al., 2017). The authors suggested that some of these disorders may have represented misdiagnosed prodromal symptoms of AD. This highlights the importance of proper differential diagnostics among older persons and the importance of appropriate time window in psychiatric and neuroepidemiology research (Tapiainen et al., 2017).

Despite converging evidence for the relationship between depression and dementia, the neurobiology of depressive symptoms in preclinical dementia syndromes has not been clearly established. According to one hypothesis, which enjoys some empirical support, clinically significant symptoms of major depressive disorder and subclinical depressive symptoms, in preclinical and prodromal $\mathrm{AD}$, may indicate $\mathrm{AD}$ pathological changes in selectively vulnerable brain regions. In $\mathrm{CN}$ older adults, clinically significant depressive symptoms have been associated with $\mathrm{AD}$-related changes: thinning of the entorhinal cortex, hippocampal volume reduction, and decreased cerebrospinal fluid (CSF) amyloid-beta 42 (Ballmaier et al., 2008; Gerritsen et al., 2011; Pomara et al., 2012). A cross-sectional study in late life major depression found reduced hippocampal volume in depressed versus control participants but no differences in cortical amyloid (measured in vivo by positron emission tomography (PET) imaging) (De Winter et al., 2016). In the aforementioned ADNI sample of participants with SSD and MCI, frontal and anterior cingulate atrophy was associated with global cognitive decline. These regions were postulated to govern cognitive decline in this highly vulnerable population (Gonzales et al., 2017).

Studies of subclinical depressive symptoms in $\mathrm{CN}$ older adults and across the $\mathrm{AD}$ spectrum have similarly shown associations with underlying structural and functional changes and more variably, with $\mathrm{AD}$ proteinopathies, A-beta and tau (Donovan et al., 2015; Babulal et al., 2016; Krell-Roesch et al., 2016; McCutcheon et al., 2016; Gatchel et al., 2017). Donovan and colleagues, in a cross-sectional study of $\mathrm{CN}$ older adults, found that subclinical depressive symptoms on the Geriatric depression scale (GDS) were associated with lower hippocampal volume, while GDS symptom clusters grouping with dysphoria, apathy, and anhedonia were associated with decreased hippocampal volume and reduced cerebral 18F-fluorodeoxyglucose (FDG) metabolism (for apathy-anhedonia symptoms); these associations were independent of cortical amyloid burden (Donovan et al., 2015). Similarly, Babulal and colleagues, assessing subclinical positive and negative affective symptoms by the GDS and other measures, found a longitudinal but not cross-sectional association between GDS and CSF and amyloid (Pittsburgh Compound B (PiB)) PET AD biomarkers (Babulal et al., 2016). In contrast, clinicopathologic studies in mixed populations of $\mathrm{CN}$ and AD participants (Wilson et al., 2003, 2014) and in MCI and mild AD participants (McCutcheon et al., 2016) have not shown robust relationships between depressive symptoms and amyloid plaques or neurofibrillary tangles (Wilson et al., 2003; Wilson et al., 2014; McCutcheon et al., 2016). Together, these findings suggest that the neurobiology of depressive symptoms may differ based on depressive symptom severity and disease stage, and may not be mediated solely by $\mathrm{AD}$ proteinopathies.

In addition to $\mathrm{AD}$, converging evidence supports depression as a risk factor and/or prodrome for vascular dementia (Barnes et al., 2012; Lin et al., 2017). Depressive symptoms may be more common in vascular dementia than in $\mathrm{AD}$ dementia (O'Brien, 2003), and depression has been associated with amnestic (Steenland et al., 2012; Geda et al., 2014) as well as both amnestic and non-amnestic MCI (Hermida et al., 2012). The robust relationship between cerebrovascular disease and depressive symptoms may underlie this association (Alexopoulos et al., 1997; Krishnan et al., 1997). In particular, disruption of frontosubcortical circuits, whether by vascular lesions or in FTD, is one mechanism hypothesized to underlie the association between depression and vascular and FTD pre-dementia syndromes.

\section{Anxiety}

There has been a growing body of research supporting an association between anxiety and reduced cognitive function in older adults, indicating clinically elevated anxiety symptoms to be associated with poorer global cognition, episodic memory, and executive functioning (Beaudreau and O'Hara, 2008). Anxiety symptoms are commonly associated with neurodegenerative diseases, and prevalence estimates range between $8 \%$ and $71 \%$ (Seignourel et al., 2008). Clinically elevated anxiety has also 
been linked to cognitive decline in older adults (Sinoff and Werner, 2003; DeLuca et al., 2005; Gallacher et al., 2009), leading some to conclude that anxiety symptoms may predict cognitive decline in older adults without dementia (Pietrzak et al., 2012). Anxiety symptoms are common in MCI (Apostolova and Cummings, 2008; Spalletta et al., 2010), and co-morbid presentation of anxiety in MCI has been shown to increase the risk of progressing to AD (Palmer et al., 2007; Palmer et al., 2010; Rabins et al., 2013; Mah et al., 2015).

Systematic reviews and meta-analyses are less conclusive, however, with some providing support for anxiety as a risk factor for dementia (Gulpers et al., 2016), while others provide mixed results (Cooper et al., 2015). For instance, Gulpers et al. (2016) conducted a systematic review and meta-analysis of 20 studies demonstrating anxiety predicts incident cognitive impairment (relative risk [RR]: $1.77,95 \%$ CI: $1.02-2.42, z=2.05, \mathrm{p}=$ 0.040 ) in the community, with greater age being a driver of results, suggesting anxiety to be a prodromal symptom. However, co-morbid anxiety in subject with MCI seen in the clinical setting did not predict conversion to dementia in this study (RR: $1.21,95 \%$ CI: $0.90-1.63, z=1.28, \mathrm{p}=0.200$ ) (Gulpers et al., 2016). Conversely, Cooper et al. (2015) provided mixed results, identifying only one higher quality epidemiological study that showed anxiety to predict AD (Palmer et al., 2007), while in three clinical studies (Robert et al., 2006; Rozzini et al., 2007; Devier et al., 2009) anxiety was found not to predict conversion (pooled odds ratio [OR] from clinical studies: $-0.11,-0.34$ to 0.11 ). Nonetheless, a recent meta-analysis by $\mathrm{Li}$ and $\mathrm{Li}$ (2017) of 11 studies showed a pooled hazard ratio of conversion to dementia for co-morbid anxiety and MCI compared to those without anxiety (hazard ratio (HR): $1.18,95 \% \mathrm{CI}: 1.07-1.31, \mathrm{p}=0.002$ ). Heterogeneity was substantial in these studies, and confounding the results is the fact that rating scales used to assess anxiety did not reliably distinguish between anxiety as part of a psychiatric illness, or anxiety as a new-onset phenomenon, with little information to determine whether psychiatric symptoms were brief and episodic, or sustained symptoms representing a change from baseline state.

While the manifestations of anxiety in prodromal patients are still under investigation, worried appearance, fearfulness, tension, restlessness and fidgeting are all common manifestations of anxiety in people with dementia (Ferretti et al., 2001). What remains unclear is the degree to which anxiety symptoms reflect a psychopathological reaction to cognitive and functional decline, or whether they arise from biological changes in emotionrelevant neural circuits (Levenson et al., 2014).
Levenson et al. (2014) conclude that there is strong evidence to support the latter, based on findings of heightened intrinsic connectivity in the salience networks in $\mathrm{AD}$ (Balthazar et al., 2013) and hypometabolism in the medial temporal lobe, superior temporal gyrus and insula (Hashimoto et al., 2006), both which have been linked to increases in anxiety. Further evidence comes from the National Alzheimer Coordinating Center (NACC) registry in a study of 2,416 CN participants over the age of 50 assessing NPS and conversion from a Clinical Dementia Rating Scale (CDR) of 0 to $>0$ (Masters et al., 2015). The Hazard Ratio of conversion in participants with anxiety was 2.83 (CI: 1.93-3.19) compared to those without anxiety. Additionally, the authors described a three-phase progression of NPS within those with cognitive decline: first, irritability, depression, and nighttime behavior changes; next, anxiety, appetite changes, agitation, and apathy; and last, elation, motor disturbances, hallucinations, delusions, and disinhibition, suggesting anxiety to be an emerging phenomenon with progressive neurodegeneration (Masters et al., 2015). Findings from the ADNI have further provided mechanistic evidence linking NPI-Q measured anxiety symptoms in amnestic MCI and conversion to AD (Mah et al., 2015). Mah et al. (2015) found that anxiety, over and beyond depression or memory decline predicted a greater decline in entorhinal cortex volume. However, it was not unequivocally determined in this study whether anxiety symptoms were causative, mediating or a consequence of neurodegeneration.

A number of potential hypotheses for a causal pathway between anxiety and cognitive impairment have been described including: (1) hypercortisolism, (2) cardiovascular disease, (3) lowgrade inflammation, (4) brain-derived neurotrophic factor suppression, and (5) depletion of cognitive reserves (as reviewed in Gulpers et al. (2016)). According to the hypercortisolism hypothesis, anxiety is a state of heightened stress and thus higher cortisol levels. Cortisol levels are in turn related to symptom severity and poorer outcomes on neuropsychological tests (Erickson et al., 2003; Leininger and Skeel, 2012; Mantella et al. 2008; Rosnick et al., 2013). Further, cortisol-induced overstimulation of glucocorticoid receptors in the medial temporal lobe have also been linked to hippocampal atrophy (Sapolsky, 2000; Erickson et al., 2003), and stress has been postulated as a mechanism for hippocampal injury and volume loss (Sheline et al., 1999). Animal studies have shown higher cortisol administration levels to increase amyloid and tau accumulation, both processes involved in AD pathology (Green et al., 2006). Anxiety has also been linked to coronary 
artery disease and stroke, with anxiety implicated in the triggering of physiological reactions (e.g. increased heart rate, blood pressure, vasoconstriction, and platelet activity) that are associated with cardiovascular disease and ultimately vascular dementia (Sheps and Sheffield, 2001; Lambiase et al., 2014; Batelaan et al., 2016). Chronic low-level inflammation is another related causal hypothesis, in which elevated cytokines such as interleukin-6 and tumor necrosis factor are observed in stress-related states such as anxiety, and in turn shown to be linked to negative effects on cognitive functioning (Furtado and Katzman (2015); Reichenberg et al., 2001). Furthermore, anxiety disorders have also been linked to decreased brain derived neurotrophic factor (BDNF) levels or polymorphisms (Domingos da Silveira da Luz et al., 2013). BDNF is needed for synaptic plasticity, learning and memory, and neuronal repair and is decreased in $\mathrm{AD}$ and MCI (Teixeira et al., 2010). BDNF signaling is necessary for antidepressant response, and BDNF deletion attenuates this response (Castrén and Kojima, 2017). The Framingham Heart Study identified higher stratified BDNF levels as a protective factor for dementia, emphasizing its importance as a marker and target (Weinstein et al., 2014). Finally, it has been proposed that chronic and recurrent anxiety disorders throughout the life-course may lead to avoidance behaviors, which in turn may result in lowered cognitive reserve as a result of less mental and social stimulation, therefore increasing the risk of dementia (Stern, 2012).

\section{Elation, euphoria, and mania}

A constellation of elation, euphoria, and mania can represent a pre-dementia syndrome. "Secondary mania," mania due to an etiology other than bipolar disorder, causes the majority of new onset mania in older adults (Krauthammer and Klerman, 1978; Brooks and Hoblyn, 2005), which include neurodegenerative disorders, such as predementia syndromes and dementias (Krauthammer and Klerman, 1978; Brooks and Hoblyn, 2005).

Evidence from cohort studies as well as retrospective data suggests that those with late onset mania are at an increased risk for development of cognitive impairment and dementia. Patients who develop their first manic episode after age 58 have higher rates of cognitive impairment, which is variably reversible (Young and Klerman, 1992; Brooks and Hoblyn, 2005). A large cohort study of 37,768 men aged $65-85$ years with bipolar disorder had an adjusted hazard ratio of 2.3 ( $\mathrm{HR}=2.30$, 95\% CI 1.80-2.94) for development of dementia
(Almeida et al., 2016). Subgroups, who developed bipolar disorder over the age of 70 or within the past five years had the greatest risk for development of dementia (Almeida et al., 2016), very similar to the evidence for depressive symptoms (Almeida et al., 2017). A retrospective case series of patients over the age of 65 admitted for mania, found 22 of 92 (24\%) had evidence of cerebral organic impairment (Stone, 1989; Almeida et al., 2016). Another case series showed that 8 of 25 (32\%) older adults with mania went on to develop cognitive impairment within 5-7 years (Dhingra and Rabins, 1991).

$\mathrm{CN}$ individuals with symptoms of elation may be at elevated risk of developing AD. A survival analysis of $11,453 \mathrm{CN}$ individuals from the NACC database showed that positive $\varepsilon 4$ carrier status and symptoms of elation, among other NPS, conferred greater risk of development of $\mathrm{AD}$ (Burke et al., 2016). Another NACC database study showed those with amnestic MCI had higher rates of elation and aggression as compared to non-amnestic MCI (Apostolova et al., 2014). However, symptoms of elation, euphoria, and mania are relatively rare in $\mathrm{AD}$ type dementia as compared to FTD, with an estimated prevalence of 2.2-3.5\% (Burns, 1992; Lyketsos et al., 1995).

The pathophysiology of late onset bipolar disorder is thought to be different from early onset bipolar disorder with the late onset group having higher rates of neurologic and vascular brain abnormalities (Young and Klerman, 1992; Tohen et al., 1994; Fujikawa et al., 1995; Sami et al., 2015). Vascular mania has been suggested as a subtype of secondary mania, when it occurs in the presence of co-morbid cerebrovascular disease or cognitive impairment (Steffens and Krishnan, 1998; Sajatovic et al., 2015). The association of cerebrovascular disease with late onset mania implies a possible prodrome to vascular dementia. Lesions in a number of different brain regions have been associated with the development of secondary mania, including bilateral orbitofrontal, right temporoparietal, right basal and medial temporal lobe, basal ganglia, thalamic and right frontotemporal (Bakchine et al., 1989; Danel et al., 1989; Turecki et al., 1993; Robinson, 1997; Starkstein and Robinson, 1997; Brooks and Hoblyn, 2005; Cerami et al., 2011). A case control series by Ramirez-Bermudez et al. (2016) showed that when compared to healthy normal adults, a group with first onset mania after age 50 had higher rates of white matter hyperintensities in the right frontal and the left temporal brain regions. A number of scientists hypothesize that damage to the right orbitofrontal region is a unifying finding in the development of secondary mania (Starkstein and Robinson, 1997; Brooks and Hoblyn, 2005). 
Disruption to orbitofrontal neurocircuitry appears common to both to secondary mania and bvFTD. More recently, two cases of sporadic FTLD with the same progranulin mutation and one case of FTLD with a C9ORF72 gene hexanucleotide expansion mutation developed symptomatology meeting criteria for a bipolar disorder diagnosis prior to clinical presentation of Frontotemporal lobar degeneration (FTLD) (Cerami et al., 2011; Floris et al., 2013). There have also been case reports of bipolar disorder mimicking BvFTD, with failure to progress to "probable" BvFTD after 3-7 years of observation (Dols et al., 2016).

\section{Irritability}

Irritability is common among neurobehavioral symptoms in neurodegenerative disease and causes increased burden on patients, caregivers, and the community alike (Sousa et al., 2016). Among cognitively impaired individuals without dementia in the Cache County cohort, irritability/lability was, among all NPI domains, second only to depression/dysphoria in prevalence (Peters et al., 2013). Irritability was the third most prevalent NPS regardless of dementia in a populationbased longitudinal study of aging conducted in England and Wales, with sleep problems more prevalent in people without dementia and apathy more prevalent in people with dementia (Savva et al., 2009). It has now been shown that irritability is among the neurobehavioral symptoms which portends more rapid decline in several datasets including NACC (Leoutsakos et al., 2015; Forrester et al., 2016); the Spanish ZARADEMP cohort (Lobo et al., 2008) and the Mayo Clinic Study of aging (Geda et al., 2014). Hence, as with other emergent behavioral symptoms, monitoring emergent irritability in the clinic would seem of the utmost importance.

Whether emergent irritability observed in the dementia prodrome should be regarded as a manifestation of a mood disturbance or one of behavioral dysregulation/disinhibition is unclear phenomenologically as there is support for both possibilities from cluster analyses (Leoutsakos et al., 2015). It might be hoped that studies of the neurobiology of irritability would ultimately serve to clarify this question thereby offering improved treatment strategies. However, such studies have suffered from this ambiguous phenomenology as well. Disinhibition together with emotional lability in dementia has been associated with orbitofrontalsubcortical circuit dysfunction (Tascone and Bottino, 2013). The same can be said when irritability falls within agitation in $\mathrm{AD}$. i.e. even with $\mathrm{AD}$, associations have been made with deficits in frontal, anterior cingulate, and posterior cingulate cortices as well as the amygdala and hippocampus (Rosenberg et al., 2015). A specific association between irritability on the NPI scale has been made with lower fractional anisotropy of the anterior cingulate in a sample of MCI and AD patients (Tighe et al., 2012). On the other hand, when irritability is grouped with affective symptoms (depression) or psychosis, no clear evidence for specific neurobiological substrates emerges (Tascone and Bottino, 2013). Recently, however, an FDG-PET study in AD demonstrated that irritability had common metabolic changes to agitation (right temporal, right frontal, bilateral middle, and posterior cingulate gyri) but differed in specific regions (right insular, precentral, and postcentral gyri) (Weissberger et al., 2017), reflecting neurodegeneration in regions associated with core $\mathrm{AD}$ pathology (Rosenberg, 2017). It is noteworthy that to date, most of the work on the neurobiology of irritability comes from the dementia literature with little data addressing emergent irritability in the predementia stages in spite of its prevalence. That said, a very recent ADNI study of biomarker positive preclinical AD participants (amyloid and tau positive, MMSE $\sim 29$,) assessed relationships between NPS and two-year interval change in metabolism measured by FDG-PET. Sleep behavior and irritability predicted posterior cingulate hypometabolism at two years, "supporting the emerging conceptual framework in which NPS constitute an early clinical manifestation of $\mathrm{AD}$ pathophysiology" ( $\mathrm{Ng}$ et al., 2017).

\section{Conclusions/Future directions}

Symptoms of affective and emotional dysregulation, ranging from depression and dysphoria, anxiety and worry, to elation, euphoria and irritability, are common in preclinical and prodromal dementia syndromes, and are often harbingers of progressive cognitive decline (Pietrzak et al., 2012; Donovan et al., 2014; Geda et al., 2014; Ismail et al., 2016; Almeida et al., 2017; Gonzales et al., 2017; Singh-Manoux et al., 2017; Tapiainen et al., 2017). This spectrum of symptoms is associated with functional impairment and decreased cognitive and psychosocial function (Karttunen et al., 2011), and has prognostic utility in $\mathrm{CN}$ older adults and in MCI for several dementia sub-types (Barnes et al., 2012; Steenland et al., 2012; Geda et al., 2014). It remains important to clarify, however, whether the affective and emotional dysregulation symptoms 
have quantitative (i.e. severity of symptoms) versus qualitative (i.e. a particular pattern of symptoms) relationships to dementia risk and progression. In an ADNI study of SSD, the chronic SSD group exhibited accelerated decline on measures of global cognition, memory, processing speed, and semantic fluency, as well as accelerated frontal lobe and anterior cingulate atrophy, compared to the nondepressed group (Gonzales et al., 2017). Thus, in this sample chronicity of low-grade symptoms, as opposed to severity, was associated with cognitive decline.

However, DSM (and the aforementioned ADNI Gonzales et al. study) are silent on natural history of symptoms (i.e. chronic vs. new-onset), and the signal may thus be muddied by a heterogeneous population with varied symptom natural history. The MBI construct is predicated on the emergence of symptoms in later life as distinct and discrete from chronic and recurrent psychiatric illness with recurrent late life episodes. Overall, we believe that context is required to determine if symptom pattern or symptom severity (or both) reflect underlying neurobiology. For example, the recent paper by Almeida et al. (2017) described a graded association between severity of depressive symptoms and the risk of dementia, but that depressive symptoms were often a prodromal manifestation of dementia, as opposed to a chronic, recurrent depressive syndrome. In contrast, Rosenberg et al. (2013) determined that severity was not a predictor of cognitive decline in the NACC cohort. Further studies in this area are required that emphasize the age of onset, severity, and qualitative pattern of symptoms.

The neurobiology of affective symptoms in pre-dementia syndromes is varied and poorly understood. Converging data point toward underlying changes in brain structure and function in selectively vulnerable regions, and accumulation of disease specific pathology (i.e. proteinopathies and/or cerebrovascular lesions) (Ballmaier et al., 2008; Gerritsen et al., 2011; Pomara et al., 2012; Donovan et al., 2015; Babulal et al., 2016; Krell-Roesch et al., 2016). Previous work has largely involved investigating the neurobiology and prognostic utility of affective symptoms in the context of standard psychiatric diagnostic criteria, rather than study of endophenotypes and clusters of symptoms. This is in part due to the lack, until recently, of rating scales to capture early mild behavioral disturbances. As demonstrated in this review, the lack of adequate measures to assess symptoms of anxiety, depression and dysphoria, euphoria, and irritability in preclinical disease states and that specify late life onset and persistence has impeded conclusive evidence with regards to the role of these NPS as a risk factor for cognitive impairment and dementia. Certainly, robust evidence from several large longitudinal and case-control epidemiological studies has highlighted the need for a determination of the age of onset of symptoms and of the timewindow between onset of symptoms and decline in cognition to properly ascertain risk (Almeida et al., 2017; Singh-Manoux et al., 2017; Tapiainen et al., 2017). The Mild Behavioral Impairment Checklist (MBI-C) (Ismail et al., 2017a) (available at www.MBItest.org) has specifically been developed to address this need, in order to systematically study NPS in pre-clinical states to determine their prognostic utility for cognitive decline and dementia.

Diverse symptoms of affect and emotional dysregulation commonly co-occur in older adults and can be challenging to systematically describe outside of the context of classic psychiatric diagnostic framework and phenomenology. Both the emotional dysregulation domain and the MBI-C as a whole comprehensively capture this spectrum of symptoms and thus provide an operationalized way to assess and track them (Ismail et al., 2017a). Further, the structure of the checklist provides the opportunity to differentiate the prognostic utility of this domain as whole as well specific symptoms within it such as anhedonia, dysphoria, sadness, and anxiety (Ismail et al., 2017a).

Adding to this complexity, the neurobiology of symptoms of affective and emotional dysregulation may differ in $\mathrm{CN}$ older adults compared to MCI, based on severity of the affective symptoms and the specific pre-dementia syndrome, and also in cases where symptoms are superimposed on a life-long recurrent affective or anxiety disorder. Future prospective studies of emotional dysregulation symptoms using the MBI framework in the clinical scenarios above are needed to further disentangle their prognostic utility and neurobiology. Studies will need to distinguish NPS reflecting core "typical" mechanisms of neurodegeneration versus those reflecting novel pathways, as such distinction carries implications for treatment. If NPS are a manifestation of typical neurodegenerative pathology, then treatment would involve agents that modify that same pathology. However, if NPS are manifestations of alternative pathology, interventions will be required for those alternative pathologies to modify outcome and course. The inconsistencies in the evidence stem, in part, from the inconsistencies in assessing natural history of symptoms i.e. chronic and recurring NPS versus new onset or emerging NPS. As the evidence base grows, the field will be able to better determine mechanisms, and distinguish between 
these two groups and their prognostic differences (if any). In addition to mechanistic studies, we suspect that intervention studies will concurrently illuminate this area, and provide further evidence on the mechanisms above.

Overall, while study and understanding of affective symptoms in MBI remains in early stages (say, compared to the understanding of MCI); new evidence is constantly emerging and informing the field that has importance implications for prevention and treatment. For example, the aforementioned paper by Almeida et al. (2017) describes a lack of antidepressant effect to decrease dementia incidence. In contrast, the paper in Neurology by Lu et al. (2009) describes donepezil delaying progression from MCI to dementia in MCI participants with depressive symptoms. However, if treating NPS is the same as treating $\mathrm{AD}$, then why are participants with significant NPS excluded from dementia clinical trials? In fact, should older adults with NPS be preferentially screened for dementia, and included in disease modifying trials? More studies are required to answer these questions, and the binary division into NPS versus dementia is likely inadequate, requiring additional variables to ascertain risk and suitability for trial enrolment, including the natural history and age of onset of NPS, as well as genetic risk.

In summary, symptoms of affective and emotional dysregulation are common harbingers of neurodegenerative change and progressive decline in several pre-dementia syndromes. Despite this, there are limited interventions to prevent and treat these symptoms and the ensuing dementia syndromes. Further, detailed study of emotional dysregulation symptom domains with the MBIC framework is critical for not only increased understanding of neurobiology, but also toward the development of prevention and treatment strategies for at-risk individuals.

\section{Conflict of interest declaration}

Z. Ismail has received consultation/advisory board funding from Eli Lilly and Merck. M. Cantillon has had research support from Pfizer, Lilly, Lundbeck, Takeda, Novartis, Impax, Prevacus, CogRx, and the Critical Path Institute that includes FDA funding.

\section{Description of authors' roles}

All authors contributed to manuscript preparation and revisions.

\section{Acknowledgments}

Z. Ismail is funded by the Canadian Institutes of Health Research, Canadian Consortium on Neurodegeneration in Aging, and The Hotchkiss Brain Institute with support from the Alzheimer Society Calgary. J. Gatchel is supported by the BrightFocus Foundation, the Alzheimer's Association, the Rogers Family Foundation, and the Harvard Medical School Department of Psychiatry Dupont Warren and Livingston Fellowships. M.E. Mortby is supported by the Australian National Health and Medical Research Council (NHMRC) and Australian Research Council (ARC) Dementia Research Development Fellowship \#1102028. The authors would like to acknowledge the Alzheimer's Association and the International Society to Advance Alzheimer's Research and Treatment (ISTAART), Neuropsychiatric Symptom Professional Interest Area, for facilitating collaboration amongst the authors.

\section{References}

Alexopoulos, G. S. et al. (1997). Vascular depression' hypothesis. Archives of General Psychiatry, 54, 915-922.

Almeida, O. P., McCaul, K., Hankey, G. J., Yeap, B. B., Golledge, J. and Flicker, L. (2016). Risk of dementia and death in community-dwelling older men with bipolar disorder. British fournal of Psychiatry, 209, 121-126.

Almeida, O., Hankey, G., Yeap, B., Golledge, J. and Flicker, L. (2017). Depression as a modifiable factor to decrease the risk of dementia. Translational Psychiatry, 7, e1117, doi:10.1038/tp.2017.90.

Apostolova, L. G. et al. (2014). Risk factors for behavioral abnormalities in mild cognitive impairment and mild Alzheimer's disease. Dementia and Geriatric Cognitive Disorders , 37, 315-326.

Apostolova, L. G. and Cummings, J. L. (2008). Neuropsychiatric manifestations in mild cognitive impairment: a systematic review of the literature. Dementia and Geriatric Cognitive Disorders, 25, 115-126.

Babulal, G. M. et al. (2016). Mood changes in cognitively normal older adults are linked to alzheimer disease biomarker levels. The American fournal of Geriatric Psychiatry, 24, 1095-1104.

Bakchine, S., Lacomblez, L., Benoit, N., Parisot, D., Chain, F. and Lhermitte, F. (1989). Manic-like state after bilateral orbitofrontal and right temporoparietal injury: efficacy of clonidine. Neurology, 39, 777-781.

Ballmaier, M. et al. (2008). Hippocampal morphology and distinguishing late-onset from early-onset elderly depression. American fournal of Psychiatry, 165, 229-237.

Balthazar, M. L. et al. (2013). Neuropsychiatric symptoms in Alzheimer's disease are related to functional connectivity alterations in the salience network. Hum Brain Mapp, 35, 1237-1246.

Barnes, D. E., Yaffe, K., Byers, A. L., McCormick, M., Schaefer, C. and Whitmer, R. A. (2012). Midlife vs 
late-life depressive symptoms and risk of dementia: differential effects for Alzheimer disease and vascular dementia. Archives of General Psychiatry, 69, 493498.

Batelaan, N. M., Seldenrijk, A., Bot, M., van Balkom, A. J. L. M. and Penninx, B. W. J. H. (2016). Anxiety and new onset of cardiovascular disease: critical review and meta-analysis. The British fournal of Psychiatry, 208, 223-231.

Beaudreau, S. A. and O'Hara, R. (2008). Late-life anxiety and cognitive impairment: a review. American fournal of Geriatric Psychiatry, 16, 790-803.

Brooks, J. O., 3rd and Hoblyn, J. C. (2005). Secondary mania in older adults. American fournal of Psychiatry, 162, 2033-2038.

Burke, S. L., Maramaldi, P., Cadet, T. and Kukull, W. (2016). Neuropsychiatric symptoms and Apolipoprotein E: associations with eventual Alzheimer's disease development. Archives of Gerontology and Geriatrics, 65, 231-238.

Burns, A. (1992). Psychiatric phenomena in dementia of the Alzheimer type. International Psychogeriatrics, 4(Suppl. 1), S43-S54.

Castrén, E. and Kojima, M. (2017). Brain-derived neurotrophic factor in mood disorders and antidepressant treatments. Neurobiology of Disease, 97, 119-126.

Cerami, C., Marcone, A., Galimberti, D., Villa, C., Scarpini, E. and Cappa, S. F. (2011). From genotype to phenotype: two cases of genetic frontotemporal lobar degeneration with premorbid bipolar disorder. Fournal of Alzheimer's Disease, 27, 791-797.

Cerejeira, J., Lagarto, L. and Mukaetova-Ladinska, E. B. (2012). Behavioral and psychological symptoms of dementia. Frontiers of Neurology, 3, 73.

Cooper, C., Sommerlad, A., Lyketsos, C. G. and Livingston, G. (2015). Modifiable predictors of dementia in mild cognitive impairment: a systematic review and meta-analysis. American fournal of Psychiatry, 172, 323-334.

Danel, T. et al. (1989). [Mood disorders and right hemisphere infarction]. Encephale, 15, 549-553.

De Winter, F.-L. et al. (2016). No association of lower hippocampal volume with alzheimer's disease pathology in late-life depression. American fournal of Psychiatry, doi: 10.1176/appi.ajp.2016.16030319.

DeLuca, A. K. et al. (2005). Comorbid anxiety disorder in late life depression: association with memory decline over four years. International fournal of Geriatric Psychiatry, 20, 848-854.

Devier, D. J. et al. (2009). The impact of anxiety on conversion from mild cognitive impairment to Alzheimer's disease. International fournal of Geriatric Psychiatry, 24, 1335-1342.

Dhingra, U. and Rabins, P. V. (1991). Mania in the elderly: a 5-7 year follow-up. Fournal of the American Geriatrics Society, 39, 581-583.

Dols, A., Krudop, W., Moller, C., Shulman, K., Sajatovic, M. and Pijnenburg, Y. A. (2016). Late life bipolar disorder evolving into frontotemporal dementia mimic. Neuropsychiatric Disease and Treatment, 12, 2207-2212.
Domingos da Silveira da Luz, A. C. et al. (2013). Translational findings on brain-derived neurotrophic factor and anxiety: contributions from basic research to clinical practice. Neuropsychobiology, 68, 129-138.

Donovan, N. J. et al. (2014). Subjective cognitive concerns and neuropsychiatric predictors of progression to the early clinical stages of alzheimer disease. The American fournal of Geriatric Psychiatry, 22, 1642-1651.

Donovan, N. J. et al. (2015). Depressive symptoms and biomarkers of Alzheimer's disease in cognitively normal older adults. Fournal of Alzheimer's Disease, 46, 63-73.

Erickson, K., Drevets, W. and Schulkin, J. (2003). Glucocorticoid regulation of diverse cognitive functions in normal and pathological emotional states. Neuroscience $\mathcal{E}$ Biobehavioral Reviews, 27, 233-246.

Ferretti, L., McCurry, S. M., Logsdon, R., Gibbons, L. and Teri, L. (2001). Anxiety and Alzheimer's disease. fournal of Geriatric Psychiatry and Neurology, 14, 52-58.

Floris, G. et al. (2013). Bipolar affective disorder preceding frontotemporal dementia in a patient with C9ORF72 mutation: is there a genetic link between these two disorders?. Fournal of Neurology, 260, 1155-1157.

Forrester, S. N., Gallo, J. J., Smith, G. S. and Leoutsakos, J. M. (2016). Patterns of neuropsychiatric symptoms in mild cognitive impairment and risk of dementia. American Fournal of Geriatric Psychiatry, 24, 117-125.

Fujikawa, T., Yamawaki, S. and Touhouda, Y. (1995). Silent cerebral infarctions in patients with late-onset mania. Stroke, 26, 946-949.

Furtado, M. and Katzman, M. A. (2015) Neuroinflammatory pathways in anxiety, posttraumatic stress, and obsessive compulsive disorders. Psychiatry Research, 229, 37-48.

Gallacher, J. et al. (2009). Does anxiety affect risk of dementia? findings from the Caerphilly prospective study. Psychosomatic Medicine, 71, 659-666.

Gatchel, J. R. et al. (2017). Depressive symptoms and tau accumulation in the inferior temporal lobe and entorhinal cortex in cognitively normal older adults: a pilot study. Fournal of Alzheimer's Disease, 59, 975-985.

Geda, Y. E. et al. (2014). Baseline neuropsychiatric symptoms and the risk of incident mild cognitive impairment: a population-based study. American fournal of Psychiatry, 171, 572-581.

Gerritsen, L., Comijs, H. C., van der Graaf, Y., Knoops, A. J., Penninx, B. W. and Geerlings, M. I. (2011). Depression, hypothalamic pituitary adrenal axis, and hippocampal and entorhinal cortex volumes- the SMART Medea study. Biological Psychiatry, 70, 373-380.

Gonzales, M. M. et al. (2017). Cortical atrophy is associated with accelerated cognitive decline in mild cognitive impairment with subsyndromal depression. The American Fournal of Geriatric Psychiatry, 25, 980-991.

Green, K. N., Billings, L. M., Roozendaal, B., McGaugh, J. L. and LaFerla, F. M. (2006). Glucocorticoids increase amyloid- $\beta$ and Tau pathology in a mouse model of Alzheimer's disease. The fournal of Neuroscience, 26, 9047-9056.

Gulpers, B., Ramakers, I., Hamel, R., Kohler, S., Oude Voshaar, R. and Verhey, F. (2016). Anxiety as a predictor for cognitive decline and dementia: a systematic 
review and meta-analysis. American fournal of Geriatric Psychiatry, 24, 823-842.

Hashimoto, H. et al. (2006). Anxiety and regional cortical glucose metabolism in patients with Alzheimer's disease. Fournal of Neuropsychiatry and Clinical Neurosciences, 18, 521-528.

Hermida, A. P., McDonald, W. M., Steenland, K. and Levey, A. (2012). The association between late-life depression, mild cognitive impairment and dementia: is inflammation the missing link?. Expert Review of Neurotherapeutics, 12, 1339-1350.

Ismail, Z. et al. (2016). Neuropsychiatric symptoms as early manifestations of emergent dementia: provisional diagnostic criteria for mild behavioral impairment. Alzheimer's \& Dementia, 12, 195-202.

Ismail, Z. et al. (2017a). The Mild Behavioral Impairment Checklist (MBI-C): a rating scale for neuropsychiatric symptoms in pre-dementia populations. Fournal of Alzheimer's Disease, 56, 929-938.

Ismail, Z. et al. (2017b). Prevalence of depression in patients with mild cognitive impairment: a systematic review and meta-analysis. Fama Psychiatry, 74, 58-67.

Ismail, Z., Malick, A., Smith, E. E., Schweizer, T. and Fischer, C. (2014). Depression versus dementia: is this construct still relevant?. Neurodegenerative Disease Management, 4, 119-126.

Jalal, H., Ganesh, A., Lau, R., Lysack, J. and Ismail, Z. (2014). Cholinesterase-inhibitor associated mania: a case report and literature review. The Canadian fournal of Neurological Sciences, 41, 278-280.

Karttunen, K. et al. (2011). Neuropsychiatric symptoms and quality of life in patients with very mild and mild Alzheimer's disease. International fournal of Geriatric Psychiatry, 26, 473-482.

Krauthammer, C. and Klerman, G. L. (1978). Secondary mania: manic syndromes associated with antecedent physical illness or drugs. Archives of General Psychiatry, 35, 1333-1339.

Krell-Roesch, J. et al. (2016). FDG-PET and neuropsychiatric symptoms among cognitively normal elderly persons: the mayo clinic study of aging. Fournal of Alzheimer's Disease, 53, 1609-1616.

Krishnan, K. R., Hays, J. C. and Blazer, D. G. (1997). MRI-defined vascular depression. American fournal of Psychiatry, 154, 497-501.

Lambiase, M. J., Kubzansky, L. D. and Thurston, R. C. (2014). Prospective study of anxiety and incident stroke. Stroke, 45, 438-443.

Leininger, S. and Skeel, R. (2012). Cortisol and self-report measures of anxiety as predictors of neuropsychological performance. Archives of Clinical Neuropsychology, 27, 318-328.

Leoutsakos, J. M., Forrester, S. N., Lyketsos, C. G. and Smith, G. S. (2015). Latent classes of neuropsychiatric symptoms in NACC controls and conversion to mild cognitive impairment or dementia. Fournal of Alzheimer's Disease, 48, 483-493.

Levenson, R. W., Sturm, V. E. and Haase, C. M. (2014). Emotional and behavioral symptoms in neurodegenerative disease: a model for studying the neural bases of psychopathology. Annual Review of Clinical Psychology, 10, 581-606.
Li, X. X. and Li, Z. (2017). The impact of anxiety on the progression of mild cognitive impairment to dementia in Chinese and English data bases: a systematic review and meta-analysis. International fournal of Geriatric Psychiatry, doi:10.1002/gps.4694.

Lin, W. C., Hu, L. Y., Tsai, S. J., Yang, A. C. and Shen, C. C. (2017). Depression and the risk of vascular dementia: a population-based retrospective cohort study. International fournal of Geriatric Psychiatry, 32, 556-563.

Lobo, A. et al. (2008). Non-cognitive psychopathological symptoms assoc w MCI and AD. Neurotoxicity Research, 14, 263-272.

Lopez-Anton, R. et al. (2015). Mild cognitive impairment diagnosed with the new DSM-5 criteria: prevalence and associations with non-cognitive psychopathology. Acta Psychiatrica Scandinavica, 131, 29-39.

Lyketsos, C. G., Corazzini, K. and Steele, C. (1995). Mania in Alzheimer's disease. Fournal of Neuropsychiatry and Clinical Neurosciences, 7, 350-352.

Mah, L., Binns, M. A. and Steffens, D. C. (2015). Anxiety symptoms in amnestic mild cognitive impairment are associated with medial temporal atrophy and predict conversion to alzheimer disease. The American fournal of Geriatric Psychiatry, 23, 466-476.

Mantella, R. C. et al. (2008). Salivary cortisol is associated with diagnosis and severity of late-life generalized anxiety disorder. Psychoneuroendocrinology, 33, 773-781.

Masters, M. C., Morris, J. C. and Roe, C. M. (2015). "Noncognitive" symptoms of early Alzheimer disease a longitudinal analysis. Neurology, 84, 1-6.

McCutcheon, S. T. et al. (2016). Clinicopathological correlates of depression in early Alzheimer's disease in the NACC. International Fournal of Geriatric Psychiatry, 31, 1301-1311.

Mortby, M. E., Burns, R., Eramudugolla, R., Ismail, Z. and Anstey, K. J. (2017). Neuropsychiatric symptoms and cognitive impariment: understanding the importance of co-morbid symptoms. Fournal of Alzheimer's Disease, 1-13.

Ng, K. P. et al. (2017). Neuropsychiatric symptoms predict hypometabolism in preclinical Alzheimer disease. Neurology, 88, 1814-1821.

O'Brien, J. (2003). Behavioral symptoms in vascular cognitive impairment and vascular dementia. International Psychogeriatrics, 15, 133-138.

Palmer, K. et al. (2010). Neuropsychiatric predictors of progression from amnestic-mild cognitive impairment to Alzheimer's disease: the role of depression and apathy. Fournal of Alzheimer's Disease, 20, 175-183.

Palmer, K., Berger, A. K., Monastero, R., Winblad, B., Backman, L. and Fratiglioni, L. (2007). Predictors of progression from mild cognitive impairment to Alzheimer disease. Neurology, 68, 1596-1602.

Peters, M. E. et al. (2012). Prevalence of neuropsychiatric symptoms in CIND and its subtypes: the cache county study. The American fournal of Geriatric Psychiatry, 20, 416-424.

Peters, M. E. et al. (2013). Neuropsychiatric symptoms as risk factors for progression from CIND to dementia: the cache county study. American fournal of Geriatric Psychiatry, 21, 1116-1124.

Pietrzak, R. H. et al. (2012). Mild worry symptoms predict decline in learning and memory in healthy older adults: a 
2-year prospective cohort study. The American fournal of Geriatric Psychiatry, 20, 266-275.

Pomara, N. et al. (2012). Lower CSF amyloid beta peptides and higher F2-isoprostanes in cognitively intact elderly individuals with major depressive disorder. American Fournal of Psychiatry, 169, 523-530.

Rabins, P. V. et al. (2013). Predictors of progression to severe Alzheimer's disease in an incidence sample. Alzheimers Dementia, 9, 204-207.

Ramirez-Bermudez, J. et al. (2016). [White matter hyperintensities and cognitive function in patients with late onset mania]. Gaceta medica de Mexico, 152, 592-600.

Reichenberg, A. et al. (2001). Cytokine-associated emotional and cognitive disturbances in humans. Archives of General Psychiatry, 58, 445-452.

Robert, P. H. et al. (2006). Apathy in patients with mild cognitive impairment and the risk of developing dementia of Alzheimer's disease: a one-year follow-up study. Clinical Neurology and Neurosurgery, 108, 733-736.

Robinson, R. G. (1997). Mood disorders secondary to stroke. Seminars in Clinical Neuropsychiatry, 2, 244-251.

Rosenberg, P. B. (2017). New Insights Into Brain Mechanisms Underlying Neuropsychiatric Symptoms in Alzheimer's Disease. Elsevier.

Rosenberg, P. B., Mielke, M. M., Appleby, B. S., Oh, E. S., Geda, Y. E. and Lyketsos, C. G. (2013). The association of neuropsychiatric symptoms in MCI with incident dementia and Alzheimer disease. The American Fournal of Geriatric Psychiatry, 21, 685-695.

Rosenberg, P. B., Nowrangi, M. A. and Lyketsos, C. G. (2015). Neuropsychiatric symptoms in Alzheimer's disease: what might be associated brain circuits?. Molecular Aspects of Medicine, 43-44, 25-37.

Rosnick, C. B., Rawson, K. S., Butters, M. A. and Lenze, E. J. (2013). Association of cortisol with neuropsychological assessment in older adults with generalized anxiety disorder. Aging $\mathcal{E}$ Mental Health, 17, 432-440.

Rozzini, L. et al. (2007). Conversion of amnestic mild cognitive impairment to dementia of Alzheimer type is independent to memory deterioration. International fournal of Geriatric Psychiatry, 22, 1217-1222.

Sajatovic, M. et al. (2015). A report on older-age bipolar disorder from the international society for bipolar disorders task force. International Fournal of Bipolar Disorders, 17, 689-704.

Sami, M., Khan, H. and Nilforooshan, R. (2015). Late onset mania as an organic syndrome: a review of case reports in the literature. Fournal of Affective Disorders, 188, 226-231.

Sapolsky, R. M. (2000). Glucocorticoids and hippocampal atrophy in neuropsychiatric disorders. Archives of General Psychiatry, 57, 925-935.

Savva, G. M. et al. (2009). Prevalence, correlates and course of behavioural and psychological symptoms of dementia in the population. British fournal of Psychiatry, 194, 212-219.

Seignourel, P. J., Kunik, M. E., Snow, L., Wilson, N. and Stanley, M. (2008). Anxiety in dementia: a critical review. Clinical Psychology Review, 28, 1071-1082.

Sheline, Y. I., Sanghavi, M., Mintun, M. A. and Gado, M. H. (1999). Depression duration but not age predicts hippocampal volume loss in medically healthy women with recurrent major depression. Fournal of Neuroscience, 19, 5034-5043.

Sheps, D. S. and Sheffield, D. (2001). Depression, anxiety, and the cardiovascular system: the cardiologist's perspective. Fournal of Clinical Psychiatry, 62(Suppl. 8), 12-16; discussion 17-18.

Singh-Manoux, A. et al. (2017). Trajectories of depressive symptoms before diagnosis of dementia: a 28 -year follow-up study. FAMA Psychiatry.

Sinoff, G. and Werner, P. (2003). Anxiety disorder and accompanying subjective memory loss in the elderly as a predictor of future cognitive decline. International fournal of Geriatric Psychiatry, 18, 951-959.

Sousa, M. F., Santos, R. L., Turro-Garriga, O., Dias, R., Dourado, M. C. and Conde-Sala, J. L. (2016). Factors associated with caregiver burden: comparative study between Brazilian and Spanish caregivers of patients with Alzheimer's disease (AD). International Psychogeriatrics, 28, 1363-1374.

Spalletta, G., Musicco, M., Padovani, A., Rozzini, L., Perri, R. and Fadda, L. (2010). Neuropsychiatric symptoms and syndromes in a large cohort of newly diagnosed, untreated patients with Alzheimer disease. International fournal of Geriatric Psychiatry, 18, 1026-1035.

Starkstein, S. E. and Robinson, R. G. (1997). Mechanism of disinhibition after brain lesions. Fournal of Nervous and Mental Disease, 185, 108-114.

Steenland, K., Karnes, C., Seals, R., Carnevale, C., Hermida, A. and Levey, A. (2012). Late-life depression as a risk factor for mild cognitive impairment or Alzheimer's disease in 30 US Alzheimer's disease centers. Fournal of Alzheimer's Disease, 31, 265-275.

Steffens, D. C. and Krishnan, K. R. (1998). Structural neuroimaging and mood disorders: recent findings, implications for classification, and future directions. Biological Psychiatry, 43, 705-712.

Stern, Y. (2012). Cognitive reserve in ageing and Alzheimer's disease. Lancet Neurology, 11, 1006-1012.

Stone, K. (1989). Mania in the elderly. British fournal of Psychiatry, 155, 220-224.

Tapiainen, V., Hartikainen, S., Taipale, H., Tiihonen, J. and Tolppanen, A.-M. (2017). Hospital-treated mental and behavioral disorders and risk of Alzheimer's disease: a nationwide nested case-control study. European Psychiatry, 43, 92-98.

Taragano, F. E., Allegri, R. F., Krupitzki, H., Sarasola, D., Serrano, C. and Lyketsos, C. (2009). Mild behavioral impairment. Fournal of Clinical Psychiatry, 70, 584-592.

Tascone, L. d. S. and Bottino, C. M. d. C. (2013). Neurobiology of neuropsychiatric symtoms in Alzheimer's disease: a critical review wiht a focus on neuroimaging. Dement Neuropsychol, 7, 236-243.

Teixeira, A. L., Barbosa, I. G., Diniz, B. S. and Kummer, A. (2010). Circulating levels of brain-derived neurotrophic factor: correlation with mood, cognition and motor function. Biomarkers in Medicine, 4, 871-887.

Tighe, S. K. et al. (2012). Diffusion tensor imaging of neuropsychiatric symptoms in mild cognitive impairment and Alzheimer's dementia. Fournal of Neuropsychiatry and Clinical Neurosciences , 24, 484-488. 
Ting, C. et al. (2010). Differentiating the cognitive profile of schizophrenia from that of Alzheimer disease and depression in late life. PloS One, 5, e10151.

Tohen, M., Shulman, K. I. and Satlin, A. (1994). First-episode mania in late life. American fournal of Psychiatry, 151, 130-132.

Turecki, G., Mari Jde, J. and Del Porto, J. A. (1993). Bipolar disorder following a left basal-ganglia stroke. British Fournal of Psychiatry, 163, 690.

Weinstein, G. et al. (2014). Serum brain-derived neurotrophic factor and the risk for dementia: the Framingham heart study. $\mathcal{F} A M A$ Neurology, 71, 55-61.

Weissberger, G. H., Melrose, R. J., Narvaez, T. A., Harwood, D., Mandelkern, M. A. and Sultzer, D. L. (2017). 18 F-Fluorodeoxyglucose positron emission tomography cortical metabolic activity associated with distinct agitation behaviors in alzheimer disease. The American fournal of Geriatric Psychiatry, 25, 569-579.

Wilson, R. S. et al. (2014). Clinical-pathologic study of depressive symptoms and cognitive decline in old age. Neurology, 83, 702-709.

Wilson, R., Schneider, J., Bienias, J., Arnold, S., Evans, D. and Bennett, D. (2003). Depressive symptoms, clinical $\mathrm{AD}$, and cortical plaques and tangles in older persons. Neurology, 61, 1102-1107.

Woolley, J. D., Khan, B. K., Murthy, N. K., Miller, B. L. and Rankin, K. P. (2011). The diagnostic challenge of psychiatric symptoms in neurodegenerative disease: rates of and risk factors for prior psychiatric diagnosis in patients with early neurodegenerative disease. Fournal of Clinical Psychiatry, 72, 126-133.

Young, R. C. and Klerman, G. L. (1992). Mania in late life: focus on age at onset. American fournal of Psychiatry, 149, 867-876. 